\title{
Lattice Distortion Induced Anomalous Ferromagnetism and Electronic Structure in FCC Fe and Fe-TM (TM=Cr, Ni, Ta and Zr) alloys
}

\author{
William Yi Wang, ${ }^{1,4}, *$ Shun Li Shang, ${ }^{1}$ Yi Wang, ${ }^{1}$ Yong Jie Hu, ${ }^{1}$ Kristopher A. Darling, ${ }^{2}$ Laszlo \\ J. Kecskes, ${ }^{2}$ Suveen N. Mathaudhu, ${ }^{3,}{ }^{\#}$ Xi Dong Hui, ${ }^{4}$ and Zi-Kui Liu ${ }^{1}$ \\ ${ }^{1}$ Department of Materials Science and Engineering, The Pennsylvania State University, \\ University Park, PA 16802, USA \\ ${ }^{2}$ U.S. Army Research Laboratory, Weapons and Materials Research Directorate, RDRL-WMM- \\ B, Aberdeen Proving Ground, MD 21005, USA \\ ${ }^{3}$ Materials Science Division, U.S. Army Research Office, Research Triangle Park, NC 27709, \\ USA \\ ${ }^{4}$ State Key Laboratory for Advanced Metals and Materials, University of Science and \\ Technology Beijing, Beijing 100083, China \\ * Corresponding author: William Yi Wang (yuw129@psu.edu, Tel.: +1-814-863-9957) \\ \# Present address: Department of Mechanical Engineering, University of California, Riverside, \\ CA 92521, USA
}




\section{Graphic Abstract}

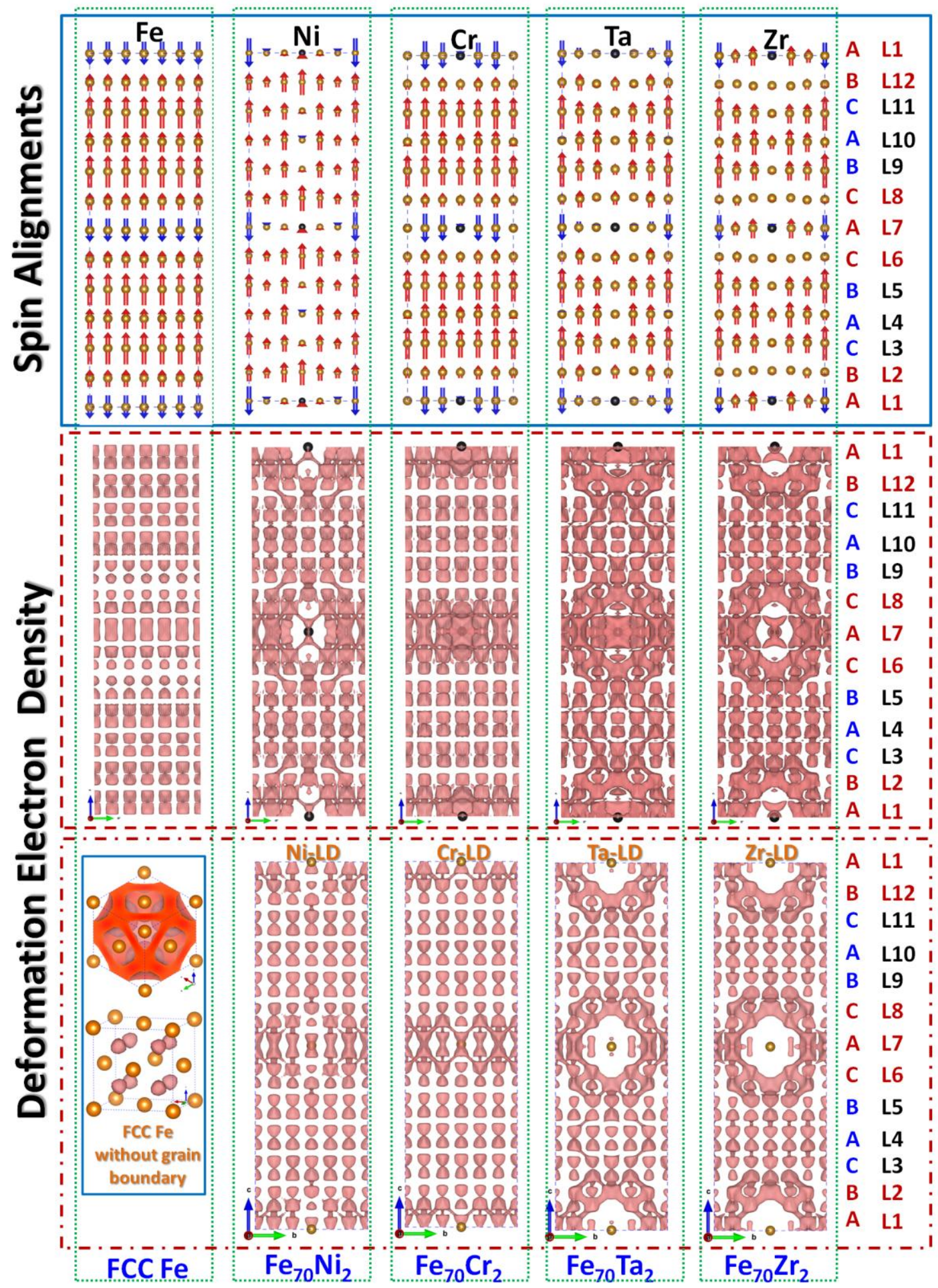




\begin{abstract}
Magnetic properties of BCC, FCC and HCP Fe and the effects of the $\sum 3<0 \overline{1} 1>\{111\}$ grain boundary (GB) and the alloying elements of $\mathrm{Cr}$, Ni, Ta and $\mathrm{Zr}$ are investigated by first-principles calculations. The FCC Fe changes continuously from the non-magnetic state at low volumes to the ferromagnetic state at high volumes. It is observed that $\Sigma 3\{111\}$ type GBs in FCC Fe have a negative formation energy since the formation of $\Sigma 3 \mathrm{~GB}$ is attributed to the local FCC-HCP transformation. Moreover, consistent with the variation of equilibrium volume caused by TM solute atoms, the magnetic moment of $\mathrm{FCC} \mathrm{Fe}_{70} \mathrm{TM}_{2}$ is decreased with alloying Ni while increased with alloying $\mathrm{Cr}$, Ta and $\mathrm{Zr}$. Due to the difference in the valence electrons and atomic radius among those solute atoms, the chemical and mechanical effects on the bond structure of $\Sigma 3\{111\} \mathrm{GB}$ in $\mathrm{Fe}$ and $\mathrm{Fe}_{70} \mathrm{TM}_{2}$ are respectively characterized by deformation electron density and plots of spin alignments. It is understood that the variation of the spin state of $\mathrm{Fe}_{70} \mathrm{TM}_{20}$ is dominated by the electron redistributions, as illustrated by the spin-flipping and the change of the bond structure. This work provides an insight into the effect of lattice distortion on ferromagnetism of $\mathrm{FCC} \mathrm{Fe}$ and $\mathrm{Fe}_{70} \mathrm{TM}_{2}$
\end{abstract}

Keywords: Magnetic materials; ab initio calculation; Electronic Structure; Defects; Crystal structure 


\section{Introduction}

The accurate description of the phase stability in Fe and Fe alloys is known to be heavily dependent on the transitions of magnetic states. Such transitions are known to be altered by electronic and lattice dynamics which are typically induced by changes in temperature and or chemistry and pressure [1-7]. For instance, at low to moderately high temperatures (0-1186K), under equilibrium conditions $\mathrm{Fe}$ is thermodynamically stabilized in its body-centered cubic phase (BCC or alpha iron lattice parameter $2.83 \AA$, vol/atom $=11.36 \AA^{3} /$ atom $[8,9]$ ) and is ferromagnetic, becoming paramagnetic (also known as the beta iron) at higher temperatures > $1043 \mathrm{~K}$. Upon increasing temperature further Fe undergoes a series of polymorphic phase transformations as the unit cell volume expands, the allotropes of which include the facecentered cubic phase (FCC or gamma iron lattice parameter $3.45 \AA$, vol/atom $=10.45 \AA^{3} /$ atom $[8$, 9]) and a high temperature (BCC or Delta iron phase lattice parameter $2.93 \AA$, vol/atom $=12.6$ $\AA^{3} /$ atom) which are stable over temperature ranges of $1183-1666 \mathrm{~K}$ and $1666-1811 \mathrm{~K}$ respectively. Additionally, at high pressures, $>15 \mathrm{GPa}$, alpha iron undergoes a transformation into the hexagonal close-packed phase (HCP or epsilon iron lattice parameters $\mathrm{a}=2.45 \AA$ and $\mathrm{c}=3.93$ $\AA ; \mathrm{c} / \mathrm{a}=1.604, \mathrm{vol} / \mathrm{atom}=10.2 \AA^{3} /$ atom $)[2,6]$. In a range of volumes near equilibrium, the ferromagnetic (FM) state is found to have the lowest energy in BCC Fe while in FCC Fe the antiferromagnetic (AFM) state has the lowest energy [10]. While magnetic states in alpha have been heavily investigated, the magnetic states of FCC Fe and Fe-based alloys have not, through they are as equally intriguing and complex [10-12] having been found to include the $2 \gamma$-state with low-spin-low-volume states (LS) and high-spin-high-volume states (HS). In addition, both the room-temperature ferromagnetism in FCC nano particles of Fe [4], and an AFM response in FCC Fe thin films ( 18̊) below 80K [13] and other structures [14, 15] have been observed. 


\begin{abstract}
The synthesis of such unique and metastable FCC Fe materials is made possible through interface constraints from the substrates due to the interplay between the free energy of phases, interfacial and strain energies.
\end{abstract}

First-principles calculations [16] have shown theoretically for some time that that the transition of FCC Fe from the non-magnetic state at low volumes to the FM state at high volumes was possible. Practically, such lattice volume distortions have been realized in the bulk, through substitutional doping or alloying $\mathrm{Fe}$ with (Al, $\mathrm{Si}, \mathrm{Ag}, \mathrm{Au}, \mathrm{Cu}, \mathrm{Ni}, \mathrm{Mn}$ ) and in the case of smaller interstitial elements $(\mathrm{C}, \mathrm{P})$ have been used stabilize/destabilize the FCC lattice $[4,11,17$ 21]. However, magnetic transitions arising from changes in volume induced by the presence of non-equlibrium defects such as grain boundaries and their chemistry has been largely ignored.

In this work, we examine the local lattice distortions caused by the formation of $\Sigma 3<1 \overline{1} 0>\{111\}$ type grain boundaries $(\mathrm{GB})$ and that caused by the segregation of selected transition metal solute atoms $(\mathrm{TM}=\mathrm{Cr}, \mathrm{Ni}, \mathrm{Ta}$ and $\mathrm{Zr}$ ) to such defects and their effects on the magnetic properties of $\mathrm{FCC} \mathrm{Fe}$ and $\mathrm{Fe}_{70} \mathrm{TM}_{2}$ alloys by means of first-principles calculations based on the density functional theory [22]. Being the most energetically favorable coincidence site lattice (CSL) GBs [23-26], $\Sigma 3<\overline{110}>\{111\}$ type GB of FCC Fe also reveal a local FCC-HCP transformation. The influence of such defects and their chemistry, on the magnetic states in $\mathrm{Fe}$ and similar materials is important in the realm of nanotechnology. Specifically, in bulk nanocrytalline metals where the total volume fraction of grain boundaries can be quite high, > $30 \%$ of the total bulk volume; the engineering of such defect states may provide insight into understanding mechanisms for preventing grain growth [27-29] or provide a path forward in synthesizing new magnetic nanocomposites or alloys. This work provides an intriguing 
explanation for how the chemical and mechanical contributions arising from defects affect the magnetic property of $\mathrm{FCC}$ and $\mathrm{Fe}_{70} \mathrm{TM}_{2}$ alloys. As a benchmark, the magnetic properties of BCC, FCC and $\mathrm{HCP} F e$ as a function of volume are investigated first. The deformation electron density and contour plots of force field are utilized to study the effects of alloying elements on the bond structure of $\Sigma 3\{111\} \mathrm{GB}$ in $\mathrm{Fe}$ and $\mathrm{Fe}_{70} \mathrm{TM}_{2}$.

\section{Methodology}

In the present first-principles calculations, the unit cell with single atom is used in the benchmark study of the magnetic states of BCC, FCC and HCP Fe. Orthorhombic supercells with 72 atoms are used to study the $\mathrm{FCC} \mathrm{Fe}$ and $\mathrm{Fe}_{70} \mathrm{TM}_{2}$ alloys with and without $\Sigma 3\{111\}$ type GBs, shown as Figure 1. It is noted that the $\Sigma 3\{111\}$ type GB can also be expressed as the FCCHCP transformation, highlighted by the red letters in Figure 1. Based on the periodic boundary condition, two solute atoms are needed, resulting in an in-plane solute concentration of 16.7 at. $\%$.

First-principles calculations at $0 \mathrm{~K}$ are carried out using the Vienna ab initio simulation package $[30,31]$ with the generalized gradient approximation (GGA) of Perdew-Wang (PW91) $[32,33]$ for the exchange-correlation functional and the projector augmented wave [34] for the electron-ion interaction. The wave functions are sampled on $\Gamma$-centered Monkhorst-Pack grids of $26 \times 26 \times 26$ for the unit cell with single atom and $10 \times 5 \times 2$ for the supercell with 72 atoms. The plane wave cutoff energy was set as $350 \mathrm{eV}$, and the energy convergence criterion of electronic self-consistency is $10^{-6} \mathrm{eV} /$ atom. While the structures are fully relaxed by the Methfessel-Paxton technique [35], the final static total energy calculations are performed by the 
tetrahedron method incorporating a Blöchl correction [36]. The ferromagnetic state of $\mathrm{Fe}_{\uparrow}$ and $\left(\mathrm{Fe}_{\uparrow}\right)_{70}\left(\mathrm{TM}_{\uparrow}\right)_{2}$ with collinear spin alignments was applied in the present calculations. The fourparameter Birch-Murnaghan equation of states $[9,37,38]$ is used to describe the relation between energy and volume. The bond structure is characterized by the deformation electron density $(\Delta \rho)$, which is defined as the electron transfer among the atoms relative to the unbonded free atoms when forming the chemical bond [39-43]. The isosurface structures with different values of $\Delta \rho$ are generated using VESTA code $[44,45]$.

The grain boundary energy, $\gamma_{\mathrm{GB}}$, is defined as [46]

Equation 1

$$
\gamma_{G B}=\frac{E_{G B}-E_{B u l k}}{2 A}
$$

where $E_{\mathrm{GB}}$ and $\mathrm{E}_{\mathrm{Bulk}}$ are the total energies of supercells with and without $\mathrm{GB}$, respectively. $\mathrm{A}$ is the grain boundary area. It is note that there are two identical GBs in the supercell based on the periodic boundary conditions. The mechanical distortion energy $\left(E_{G B}^{M}\right)[47]$ of caused by the GB is defined below can be used to investigate the contribution of lattice distortion caused by the solute atoms,

Equation 2
\[ E_{G B}^{M}=E_{G B}^{0}-E_{G B}^{\prime}(T M) \]

where $E_{G B}^{0}$ and $E_{G B}^{\prime}(T M)$ denote the total energies of Fe supercells with grain boundaries, calculated using equilibrium lattice structures obtained without and with the solute atoms, respectively. 


\section{Results and discussions}

The magnetic moments and total energies of BCC, FCC and HCP Fe are shown in Figure 2(a) as a function of volume, the equilibrium values are shown in Table I. The double-well features within the energy-volume curves of FCC and HCP Fe indicate their first-order magnetic transitions at 0 Kelvin, while the continuous energy-volume curve of BCC Fe depicts a secondorder magnetic transition. Our calculated equilibrium values of energy, equilibrium volume, bulk modulus, magnetic moments of FM BCC Fe, FM-HS FCC Fe and NM HCP Fe match well with data taken from literature, as shown in Table I. From the non-magnetic state to ferromagnetic state, $\mathrm{HCP} F e$ changes its magnetic states dramatically without an intermediate stage while FCC Fe changes gradually with FM-LS state locating in the middle of NM and FMHS. It seems the magnetism state variation of HCP Fe is more sensitive than that of FCC Fe when the volume is closes to $\sim 11.2 \AA^{3} /$ atom. Since $\Sigma 3\{111\}$ GBs can also be expressed as the FCC-HCP transformation as shown Figure 1, magnetic transitions of individual Fe allotropes shown in Figure 2(a) serve as benchmarks discussing the effect of lattice distortion caused by $\Sigma 3\{111\}$ GBs on the magnetic property of Fe. It is worth to mention that the effects of supercell size and setting in first-principles should be rigorously checked when calculating the grain boundary energy together with the variations of magnetism moment. In this work, energy, bulk modulus and magnetic moment of FCC unit cell and 48 and 72 atoms supercells compared in Table I. It can be seen that the larger the supercell size yields a better accuracy, also shown in our previous work calculating the interfacial energies of $\mathrm{Mg}$ [46]. Therefore, the supercell with 72 atoms is utilized in the study of $\mathrm{FCC} \mathrm{Fe}$ and $\mathrm{Fe}_{70} \mathrm{TM}_{2}$ alloys with and without $\Sigma 3\{111\}$ type GBs. 
It is evident from Figure 2(a-2) that HCP Fe is more stable than FCC Fe at 0 Kelvin, resulting in a negative grain boundary energy for $\Sigma 3\{111\}$ GBs as its formation represents a local FCC-HCP transformation, which is further shown in Figure 2(b) and Table II. Even though HCP Fe has an equilibrium volume smaller than that of FM-LS FCC Fe and is nonmagnetic, the HCP Fe associated with the formation of the $\Sigma 3\{111\} \mathrm{GB}$ is FM and has its volume increased beyond that of FM-LS Fe. Since the equilibrium volume of FM-LS FCC Fe is smaller than that of FM HCP Fe, the magnetic moment of FM-LS FCC Fe is increased by the formation of $\Sigma 3\{111\}$ GBs from $0.31 \mathrm{uB}$ to $0.61 \mathrm{uB}$. This is also reflected by the anomalous increase of the magnetic moment of FCC Fe with $\Sigma 3\{111\}$ GBs at a smaller volume than that without GBs.

The effects of solute atoms on the magnetic moment of the $\Sigma 3 \mathrm{~GB}$ of $\mathrm{FCC} \mathrm{Fe}_{70} \mathrm{TM}_{2}$ is shown in Figure 2(b-1) and summarized in Table II. It can be seen that the calculated equilibrium volumes of the $\mathrm{FCC} \mathrm{Fe}_{70} \mathrm{TM}_{2}$ alloys are increased by $\mathrm{Ta}$ and $\mathrm{Zr}$, decreased by $\mathrm{Ni}$, and kept nearly constant by $\mathrm{Cr}$, consistent with the tendency of the change of volume. The mechanical distortion energy defined by Equation 2 is shown in Figure 2(c-2) and listed in Table II, and Figure 2(c-1) plots the magnetic moment of FCC Fe with the lattice distortion. It can be seen that the local HCP structure in the $\Sigma 3\{111\}$ type GB is stabilized by Cr, represented by the lower energy curve shown in Figure 2(c-2) and the negative $E_{G B}^{M}$ value in Table II. The contribution of lattice distortion to stability of the local HCP structure in $\Sigma 3\{111\}$ type GB decreases in the order of $\mathrm{Cr}, \mathrm{Ni}, \mathrm{Ta}$, and $\mathrm{Zr}$, shown in Figure 2(c-2). Combining with the chemical effect caused by the different valence electrons of TM shown in Figure 2(b-1), the magnetic moment of FCC $\mathrm{Fe}_{70} \mathrm{TM}_{2}$ is smaller than that of FM-LS FCC Fe, indicating that the redistribution of electrons plays an important role in the magnetic moment of Fe. 
To further understand the aforementioned anomalous ferromagnetic moment of FCC Fe and $\mathrm{Fe}_{70} \mathrm{TM}_{2}$ alloys, lattice distortions caused by the formation of $\Sigma 3\{111\} \mathrm{GBs}$ and the segregation of solute atoms are characterized by bond length dispersions and local force field, shown in Figure 3(a). It is can be seen that there are 3 typical bond lengths in FCC Fe with $\Sigma 3\{111\} \mathrm{GB}$, associating to the FCC (overlapped solid and open stars), HCP (solid star above the others) and FCC-HCP interface (solid star below the others). Correspondingly, atoms locating at different layer $\Sigma 3\{111\} \mathrm{GB}$ of Fe have 3 different magnetic moments, including spin up with long arrows for elongated bond length (L3, L5, L9 and L11 layers) and with short arrows for normal bond length (L2, L4, L6, L7, L8 and L12 layers) and spin down (L1 and L7) for HCP, shown in Figure 3(b). The lattice distortion caused by $\Sigma 3\{111\}$ GB results in a spin-flipping at the grain boundary forming local anti-ferromagnetic state, highlighted by the arrows in blue in Figure 3(b). With the segregation of solute atoms, the bond length distribution ranges of $\mathrm{Fe}_{70} \mathrm{TM}_{2}$ are in line with the difference of atomic radius between $\mathrm{Fe}$ and $\mathrm{TM}$. In particular, $\mathrm{Zr}$ with the largest atomic radius results in the widest range of the bond length, reducing the magnetic moment of its first 3 nearest neighbors. Ni with the smallest atomic radius reduces the equilibrium volume of $\Sigma 3\{111\}$ GB thus to decrease the magnetic moment of the system, as listed in Table II. Therefore, the lattice distortions caused by solute atoms and $\Sigma 3\{111\} \mathrm{GB}$ yield an anomalous local magnetic moments. It is concluded that magnetic moment $\mathrm{Fe}$ atom is reduced by a lattice distortion with a decreased bond length (compression) and enhanced by that with an increased bond length (tensile), providing an insight into the volume dependent magnetic moment shown in Figure 2.

Based on previous works $[1,20,21,48-50]$, it is noted that with alloying the various TM solute atoms having different valence electrons, their electron redistribution range and 
tomography provide direct descriptions of the lattice distortion and the related magnetic property. For example, the lattice distortion caused by carbon creates locally a region of ferromagnetic ordering, which is attributed to the formation of strong FM bonds and the redistribution of the total Fe eDOS at Fermi level [1]. The bond strength of $\Sigma 3\{111\} \mathrm{GB}$ is weakened by the segregation of $\mathrm{P}$ and $\mathrm{Cu}$ due to the formation of the covalentlike $\mathrm{Fe}-\mathrm{P}$ and $\mathrm{Fe}-\mathrm{Cu}$ bonds [20, 21]. Therefore, a deeper insight into the solute-solvent interaction together with the lattice distortion can be captured comprehensively via studying either the Fermi surface [1, 48, 49] or the deformation electron density [40-42]. And the later one is adopted in this work since the bond morphology is displayed directly.

Figure 4 shows the chemical (alloying elements) and mechanical (lattice distortion) effects on the electron redistributions of $\mathrm{FCC} \mathrm{Fe}$ and $\mathrm{Fe}_{70} \mathrm{TM}_{2}$ characterized by deformation electron density isosurface. The $3 \mathrm{D}$ views of the $\Delta \rho=0.0034 \mathrm{e}^{-} / \AA^{3}$ isosurface display the bonding network of the unite cell of FCC Fe shown in Figure $4(\mathrm{a}-1)$ while the $\Delta \rho=0.0104 \mathrm{e}^{-} / \AA^{3}$ isosurface presents the typical tetrahedrons bond morphology of the FCC lattice [41] shown in (a-2). Referring to the $\Delta \rho=0.0104 \mathrm{e}^{-} / \AA^{3}$ isosurface shown in Figure 4(a) and the local force shown in Figure 3(b), it can be see that the tetrahedrons bond of the FCC lattice transforms into the rod-like bond of the HCP lattice (layer L7) $[39,42,51]$ due to the lattice distortion in $\Sigma 3$ $\{111\} \mathrm{GB}$, shown as Figure 4(b). Herein, the formation of the $\Sigma 3\{111\} \mathrm{GB}$ is proved to be the FCC-HCP transformation by the deformation electron density isosurface. Moreover, the rod-like bonds in the GB plane (L7) are modified by changing their bonding electron density, resulting in the different bonding strength. This is because of the Coulomb force is proportional to the charge distribution of the atoms and the bonding electron distribution [39, 41]. The large value of $\Delta \rho$ indicates the enhanced bond strength. It can be seen that the $\mathrm{Ni}$ and $\mathrm{Zr}$ have a weak local 
bond strength with their first nearest neighbor $\mathrm{Fe}$ but enhance the bond strength of their $\mathrm{Fe}-\mathrm{Fe}$ neighbors (i.e., atoms in L5-L9), highlighting the enhanced bond strength of the $\Sigma 3\{111\}$ GB. Both the $\mathrm{Cr}$ and $\mathrm{Ta}$ atoms exhibit a strong bond strength with their first nearest neighbor $\mathrm{Fe}$. However, Cr shows no long range strengthen effect while Ta displays a long range strengthen effect on the bond strength.

After replacing the TM by $\mathrm{Fe}$ in a fixed lattice, the chemical and mechanical contributions to the electron redistributions of $\Sigma 3\{111\}$ type GBs are clearly distinguished in Figure 4(b)-(f). For instance, in $\mathrm{Fe}_{70} \mathrm{Cr}_{2}$, the $\mathrm{FCC}$-type bonds are modified into Bee-net type by the lattice distortion, which are further strengthened by $\mathrm{Cr}$ atoms. Similarly, in $\mathrm{Fe}_{70} \mathrm{Ni}_{2}$, the FCC-type bonds are changed into wave type by the lattice distortion and further updated into Bee-net type with 4 dumbbells nearby. The wreath type bonds having a weakened bond strength centered locally around a singular atom is attributed to the large distortion caused by the presence of $\mathrm{Ta}$ and $\mathrm{Zr}$ atoms, shown in Figure 4(e-2) and (f-2). With the addition of $\mathrm{Ta}$ and $\mathrm{Zr}$ atoms, the bond strengthen of the solute-solvent atoms in the center of the wreath is improved, shown in Figure 4(e-1) and (f-1).

It is worth mentioning that the affected zone observed in the bond morphology by those solute atoms shown in Figure 4(c)-(f) support the details of bond length dispersion discussed in Figure 3(a). For example, although $\mathrm{Cr}$ atoms exhibit a larger atomic radius than that of $\mathrm{Ni}$, segregation at the GB results in a smaller bond length dispersion range than that induced by the presence of $\mathrm{Ni}$ atoms, this is in line with the variation of their electron redistribution zone (shown as layers L5-L9 in Figure 4(c-1) and (d-1)). Moreover, as listed in Table II, it is found that a large value of $\gamma_{\mathrm{GB}}$ of $\mathrm{Fe}-\mathrm{TM}$ is attributed to a long range in electron redistribution, which reveals the physical nature of the higher $\gamma_{\mathrm{GB}}$ of $\mathrm{Fe}-\mathrm{Ta}$ and $\mathrm{Fe}-\mathrm{Zr}$ than those of $\mathrm{Fe}-\mathrm{Cr}$ and $\mathrm{Fe}-\mathrm{Ni}$. 
To obtain a better understanding of how the magnetic properties of FCC Fe are affected by the lattice distortion, the electronic structure of $\mathrm{FCC} \mathrm{Fe}$ and $\mathrm{Fe}_{70} \mathrm{TM}_{2}$ are shown in Figure 5. The common features of the partial electron density of states (eDOS) are (i) the non-zero densities around the Fermi levels, locate in a place with a sharp gradient, indicating the stability of $\Sigma 3\{111\} \mathrm{GB}$ in ferromagnetic $\mathrm{FCC} \mathrm{Fe}$ and $\mathrm{Fe}_{70} \mathrm{TM}_{2}$ alloys; and (ii) the total net spin states (defined as the difference states between spin-up and spin down) of $\mathrm{FCC} \mathrm{Fe}_{70} \mathrm{TM}_{2}$ alloys at the Fermi energy level are maintained by adding $\mathrm{Cr}$, Ni, Ta and $\mathrm{Zr}$. On the contrary, the specific features of the total net spin states of $\mathrm{FCC} \mathrm{Fe}$ and $\mathrm{Fe}_{70} \mathrm{TM}_{2}$ alloys at the Fermi energy level caused by the formation of $\Sigma 3\{111\} \mathrm{GB}$ are (i) the total net spin states of FCC Fe is switched from down to up; (ii) the total net spin states of $\mathrm{FCC} \mathrm{Fe}_{70} \mathrm{Cr}_{2}$ and $\mathrm{Fe}_{70} \mathrm{Zr}_{2}$ are changed from down to up while those of $\mathrm{Fe}_{70} \mathrm{Ni}_{2}$ and $\mathrm{Fe}_{70} \mathrm{Ta}_{2}$ are maintained. Since the small value of the total net spin states indicate a suppressed magnetism [52], the magnetic moments of Fe atoms are improved by the lattice distortions through enhancing both the spin up and spin down states. Moreover, it is understood that the variation of the spin state of $\mathrm{Fe}_{70} \mathrm{TM}_{20}(\mathrm{TM}=\mathrm{Cr}, \mathrm{Ni}, \mathrm{Ta}$ and $\mathrm{Zr})$ is dominated by the electron redistributions, as illustrated by the spin-flipping and the change of the bond structure characterized by deformation electron density.

\section{Conclusion}

In summary, we demonstrated an anomalous magnetic transformation of $\mathrm{FCC} \mathrm{Fe}_{70} \mathrm{TM}_{2}$ $(\mathrm{TM}=\mathrm{Cr}, \mathrm{Ni}$, Ta and $\mathrm{Zr}$ ) alloys induced by the FCC-HCP type phase transformation of the $\sum 3<0 \overline{1} 1>\{111\}(\mathrm{GB})$. The continuous variations of magnetic properties of BCC, FCC and HCP Fe with increasing volume display comprehensively their transition from the low-spin (LS) /non-magnetic (NM) state at low volumes to the high-spin (HS) state at high volumes. Besides 
the change of the electron density at the Fermi level induced by lattice distortion, the bond structure characterized by the deformation electron density reveals the physical nature of atomic spin-orbital interactions, which results in the anomalous ferromagnetism of $\mathrm{FCC} \mathrm{Fe}$ and $\mathrm{Fe}_{70} \mathrm{TM}_{2}$ alloys. It is found that the $\Sigma 3\{111\}$ type GB of FCC Fe has a negative formation energy and enhances the magnetic moment since the core structure of $\Sigma 3$ GB is attributed to the local FCCHCP transformation. In line with the variation of equilibrium volume caused by the TM solute atoms, the magnetic moment of $\mathrm{FCC} \mathrm{Fe}_{70} \mathrm{TM}_{2}$ is decreased with alloying $\mathrm{Ni}$ atoms while increased with alloying $\mathrm{Cr}$, Ta and $\mathrm{Zr}$. It is understood that the variation of the spin state of $\mathrm{Fe}_{70} \mathrm{TM}_{20}$ is dominated by the electron redistributions, as illustrated by the change of the bond structure characterized by deformation electron density. 


\section{Acknowledgement}

This work was financially supported by the National Science Foundation (Grant No. DMR-1006557) and the Army Research Laboratory (W911NF-08-2-0064 and W911NF-09-20045) in the Unites States and National Natural Science Foundation of China (50431030 and 50871013). W.Y. Wang acknowledges the support from the Project Based Personnel Exchange Program with American Academic Exchange Service and China Scholarship Council (2008[3072]). First-principles calculations were carried out on the LION clusters at the Pennsylvania State University supported by the Materials Simulation Center and the Research Computing and Cyberinfrastructure unit at the Pennsylvania State University. Calculations were also carried out on the CyberStar funded by NSF through grant OCI-0821527 and the XSEDE supported by NSF with Grant No.ACI-1053575. 
Table I Total energy (E) at equilibrium volume $\left(\mathrm{V}_{0}\right)$, bulk modulus (B), and magnetic moment $(\mu)$ of NM, FM, and AFM states of BCC, FCC and HCP Fe

\begin{tabular}{|c|c|c|c|c|c|c|}
\hline Structure & \multicolumn{2}{|c|}{ States } & $\underset{\text { eV/atom }}{\mathbf{E}_{\mathbf{0}}}$ & $\begin{array}{c}\mathbf{V}_{\mathbf{0}} \\
\AA^{3} / \text { atom }\end{array}$ & $\begin{array}{c}\mathbf{B} \\
\mathrm{GPa}\end{array}$ & $\underset{\mu \mathrm{B}}{\boldsymbol{\mu}}$ \\
\hline $\mathbf{B C C}$ & \multicolumn{2}{|c|}{ FM } & $\begin{array}{l}-8.317 \\
-8.275^{a}\end{array}$ & $\begin{array}{c}11.433 \\
11.358^{b} \\
- \\
11.453^{\mathrm{d}}\end{array}$ & $\begin{array}{c}176.0^{b} \\
189.3^{b}\end{array}$ & $\begin{array}{c}2.18 \\
- \\
2.08^{\mathrm{c}} \\
2.2^{\mathrm{d}}\end{array}$ \\
\hline \multirow[t]{6}{*}{ FCC } & \multicolumn{2}{|c|}{ AFM } & $-8.187^{a}$ & $\begin{array}{c}10.230^{\mathrm{a}} \\
10.448^{\mathrm{b}} \\
- \\
10.177^{\mathrm{e}} \\
11.567^{\mathrm{f}}\end{array}$ & $194.6^{\mathrm{b}}$ & $\begin{array}{c}- \\
- \\
0.64^{\mathrm{c}} \\
1.8^{\mathrm{e}} \\
1.6^{\mathrm{f}}\end{array}$ \\
\hline & \multicolumn{2}{|c|}{ NM } & -8.064 & 9.873 & - & - \\
\hline & \multirow[t]{3}{*}{ FM-LS } & 1 atom & -8.159 & 10.482 & 166.1 & 0.98 \\
\hline & & 48 atom & -8.073 & 10.243 & 236.6 & 0.31 \\
\hline & & 72 atom & -8.076 & 10.298 & 236.7 & 0.31 \\
\hline & \multicolumn{2}{|c|}{ FM-HS } & -8.163 & $\begin{array}{c}12.030 \\
- \\
11.471^{\mathrm{e}} \\
12.974^{\mathrm{f}} \\
- \\
\end{array}$ & 165.9 & $\begin{array}{c}2.57 \\
2.47^{\mathrm{c}} \\
2.5^{\mathrm{e}} \\
2.3^{\mathrm{f}} \\
2 \pm 0.5^{\mathrm{g}} \\
\end{array}$ \\
\hline \multirow[t]{2}{*}{ HCP } & \multicolumn{2}{|c|}{ NM } & $\begin{array}{c}-8.227 \\
-8.267^{\text {a }}\end{array}$ & $\begin{array}{c}10.182 \\
10.179^{\mathrm{b}}\end{array}$ & $\begin{array}{c}289.6 \\
288.3^{b}\end{array}$ & - \\
\hline & \multicolumn{2}{|c|}{ FM } & -8.124 & 12.038 & 163.3 & 2.6 \\
\hline
\end{tabular}

Notes: LS-Low spin state; HS-High spin state. The unit cell with single atom is used for BCC and HCP in the benchmark study. Different number of atoms in the FCC unit cell and supercells are tested, in which the $\Gamma$-centered Monkhorst-Pack grids of $10 \times 5 \times 2$ is utilized for the supercell with 48 atoms.

${ }^{\mathrm{a}}$ Wang, first-principles calculations with PAW-GGA [8]

${ }^{\mathrm{b}}$ Shang, first-principles calculations with PAW-GGA [9]

${ }^{c}$ Wang, first-principles calculations with PAW-LDA [10]

${ }^{\mathrm{d}}$ Medvedeva, first-principles calculations with PAW-GGA-PBE [19]

${ }^{\mathrm{e}}$ Boukhvalov, first-principles calculations [1]

${ }^{\mathrm{f}}$ Antropov, first-principles calculations [53]

${ }^{\mathrm{g}}$ Wei, first-principles calculations with PAW-LDA and PAW-GGA together with experimental measurement by Mossbauer spectroscopy [4] 
Table II Total energy $\left(E_{0}\right)$, segregation energy $\left(E_{\text {seg }}\right)$, grain boundary energy $\left(\gamma_{\mathrm{GB}}\right)$, mechanical distortion energy $\left(E_{G B}^{M}\right)$, bulk modulus (B), and magnetic moment ( $\mu$, FM state with low-spin labeled as FM-L) of FCC Fe and Fe-TM (TM=Cr, Ni, Ta and $\mathrm{Zr}$ ) alloys with and without $\Sigma 3<1 \overline{10}>\{111\}$ grain boundary.

\begin{tabular}{|c|c|c|c|c|c|c|c|c|}
\hline System & States & $\begin{array}{c}\mathbf{E}_{\mathbf{0}} \\
\mathrm{eV} / \text { atom }\end{array}$ & $\begin{array}{c}\mathbf{E}_{\text {seg }} \\
\mathrm{meV} / \mathrm{atom}\end{array}$ & $\begin{array}{c}\gamma_{\mathbf{G B}} \\
\mathrm{mJ} / \mathrm{m}^{2}\end{array}$ & $\begin{array}{c}E_{G B}^{M} \\
\mathrm{eV}\end{array}$ & $\begin{array}{c}\mathbf{V}_{\mathbf{0}} \\
\AA^{3} / \mathrm{atom}\end{array}$ & $\begin{array}{c}\mathbf{B} \\
\mathrm{GPa}\end{array}$ & $\begin{array}{c}\boldsymbol{\mu} \\
\mu \mathrm{B}\end{array}$ \\
\hline \multirow{2}{*}{$\mathbf{F e}$} & $\mathrm{FCC}$ & -8.076 & - & - & - & 10.298 & 248.7 & 0.31 \\
\cline { 2 - 9 } & $\Sigma 3$ & -8.101 & - & -563.5 & - & 10.444 & 201.3 & 0.61 \\
\hline \multirow{2}{*}{$\mathbf{F e}-\mathbf{C r}$} & $\mathrm{FCC}$ & -8.114 & - & - & - & 10.341 & 224.5 & 0.74 \\
\cline { 2 - 9 } & $\Sigma 3$ & -8.121 & -252 & -175.3 & -0.060 & 10.341 & 258.3 & 0.50 \\
\hline \multirow{2}{*}{$\mathbf{F e}-\mathbf{N i}$} & $\mathrm{FCC}$ & -7.988 & - & - & - & 10.292 & 185.6 & 0.78 \\
\cline { 2 - 9 } & $\Sigma 3$ & -8.002 & -504 & -280.0 & 0.215 & 10.282 & 218.4 & 0.30 \\
\hline \multirow{2}{*}{$\mathbf{F e}-\mathbf{T a}$} & $\mathrm{FCC}$ & -8.170 & - & - & - & 10.506 & 264.6 & 0.51 \\
\cline { 2 - 9 } & $\Sigma 3$ & -8.174 & -144 & -49.2 & 1.127 & 10.521 & 267.0 & 0.52 \\
\hline \multirow{2}{*}{ Fe-Zr } & FCC & -8.053 & - & - & - & 10.463 & 469.74 & 0.88 \\
\cline { 2 - 9 } & $\Sigma 3$ & -8.055 & -72 & -29.5 & 1.973 & 10.537 & 313.8 & 0.62 \\
\hline
\end{tabular}

Notes: The supercell with 72 atoms is utilized in the study of $\Sigma 3<110>\{111\}$ GB . 
(a)

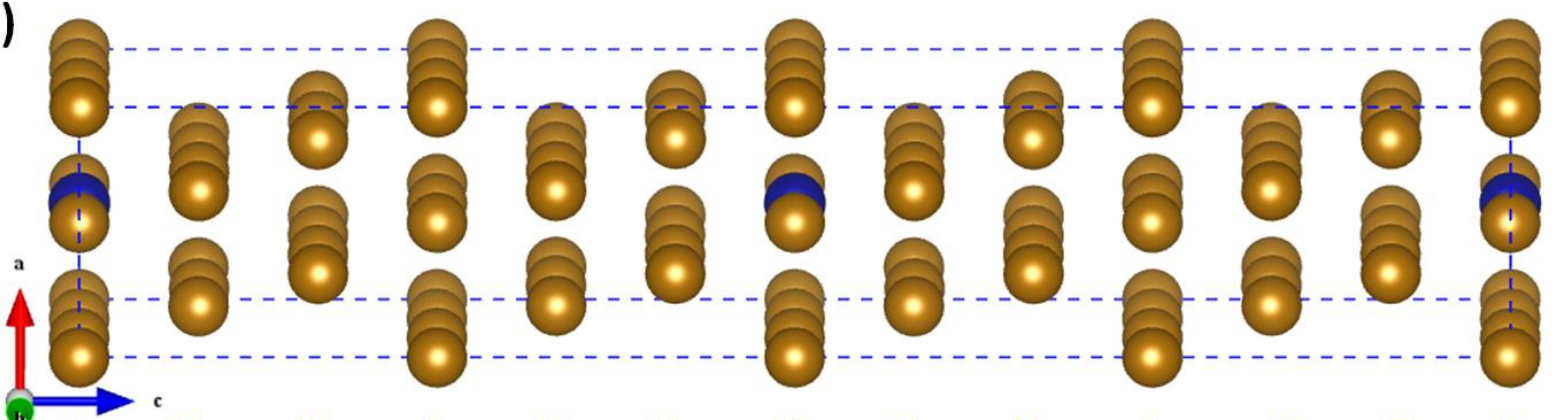

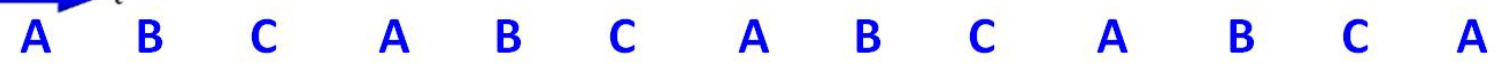
$\begin{array}{lllllllllllll}\text { L1 } & \text { L2 } & \text { L3 } & \text { L4 } & \text { L5 } & \text { L6 } & \text { L7 } & \text { L8 } & \text { L9 } & \text { L10 } & \text { L11 } & \text { L12 } & \text { L1 }\end{array}$

(b)

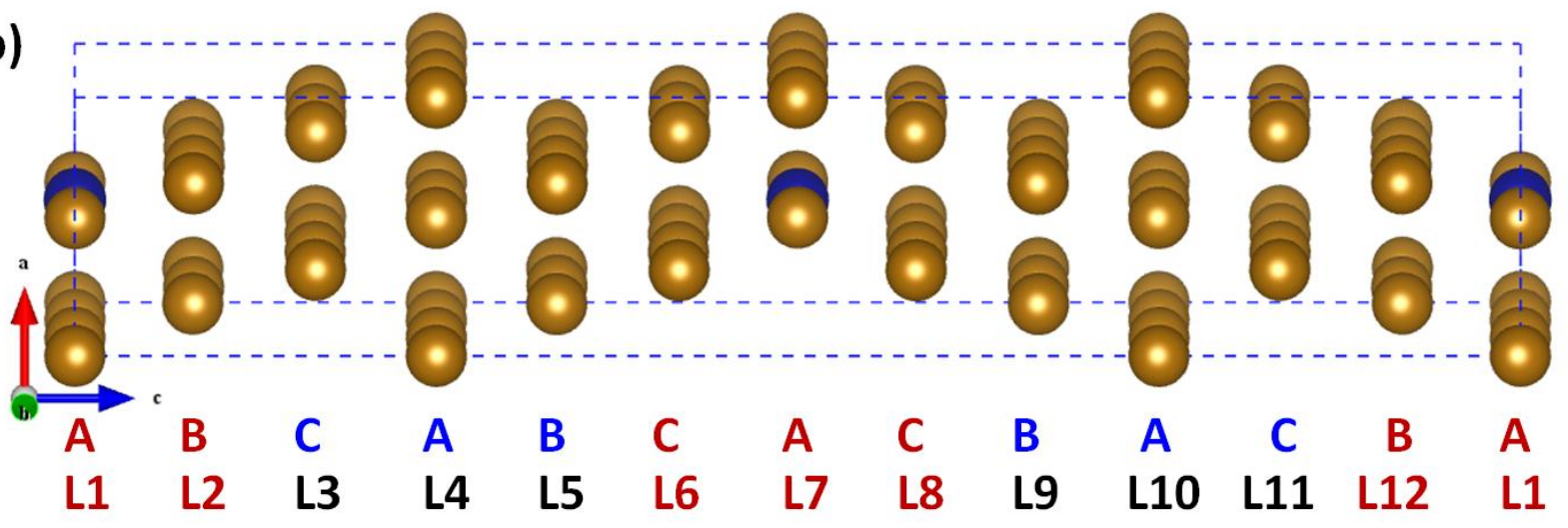

Figure 1 Schematic structures of the Fe-TM alloys, (a) FCC; and (b) $\sum \mathbf{3}<0 \overline{\mathbf{1 1}}>\{\mathbf{1 1 1}\}$. The atoms in different close packed layers are depicted with the classical FCC-type stacking sequence $(\mathrm{ABC})$ and a layer number (L\#). The blue and golden colors are applied to identify the solute atom and the matrix, respectively. The letters and the layer numbers in red mean the region of boundaries induced the local FCC-HCP transformation. 

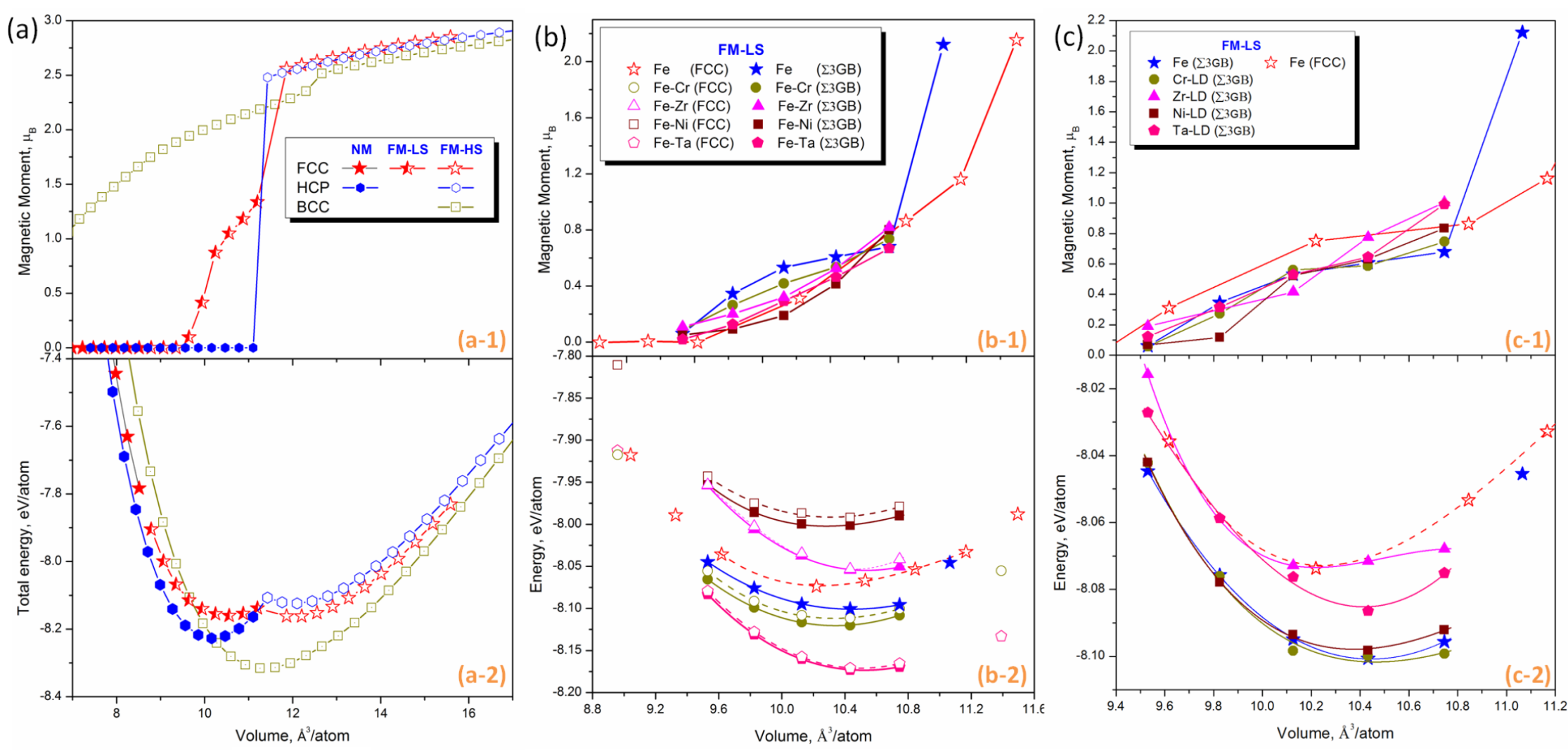

Figure 2 Graphic descriptions of magnetic states (i.e., NM - Non-magnetism, FM-LS - Ferromagnetism with low-spin, and FM-HS -

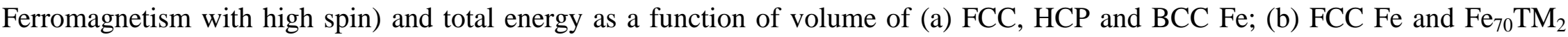
$(\mathrm{TM}=\mathrm{Cr}, \mathrm{Ni}$, Ta and $\mathrm{Zr}$ ) alloys with and without $\Sigma 3<110>\{111\}$ grain boundary in FM-LS state and (c) Contribution of lattice distortion (LD, induced by TM) to the properties of FCC Fe. 

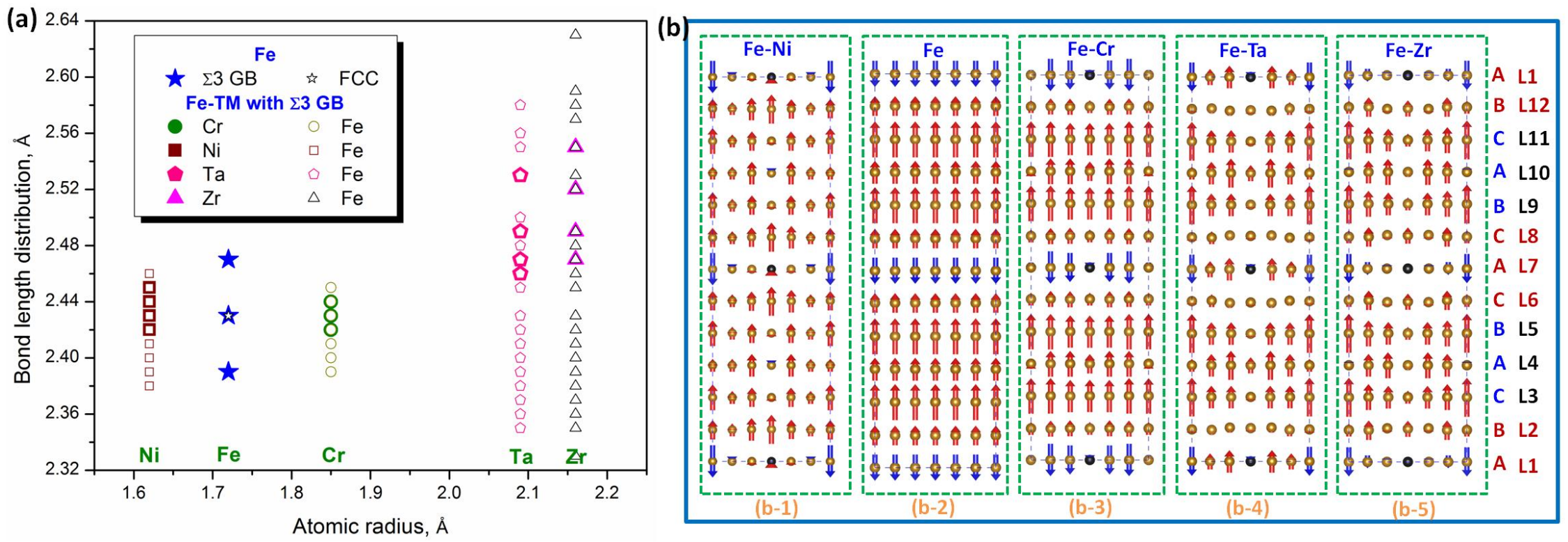

Figure 3 Impact of grain boundary and alloying elements on (a) the bond length and (b) the collinear spin alignments with two arrows.

The arrows in red and in blue identify atoms with spin up and spin down, respectively. Since the initial spins for all atoms are up, the spin-flipping caused by lattice distortions is highlighted by the arrows in blue. The length of the arrow qualitatively shows the value of magnetic moment of each atom (in $\mathrm{u}_{\mathrm{B}}$ unit). 

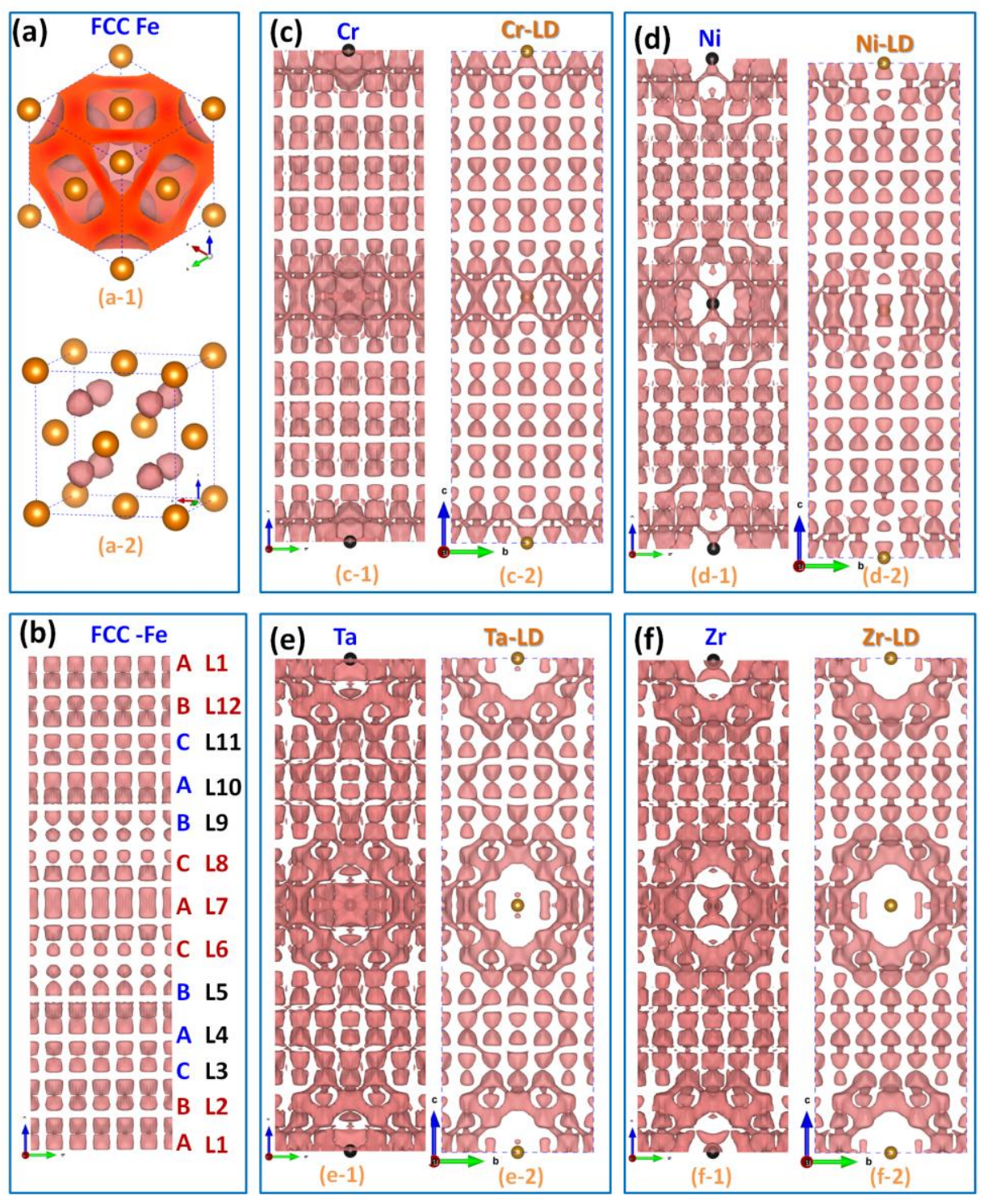

Figure 4 Deformation electron density isosurface of $\mathrm{FCC} \mathrm{Fe}$ and $\mathrm{Fe}_{70} \mathrm{TM}_{2}(\mathrm{TM}=\mathrm{Cr}, \mathrm{Ni}$, Ta and $\mathrm{Zr}$ ), (a) 3D views of the $\Delta \rho=0.0034 \mathrm{e}^{-} / \AA^{3}$ (a-1) and $\Delta \rho=0.0104 \mathrm{e}^{-} / \AA^{3}$ (a-2) isosurface of the unite cell of FCC Fe; and (b)-(f) the $\Delta \rho=0.0104 \mathrm{e}^{-} / \AA^{3}$ isosurface of $\Sigma 3<1 \overline{10}>\{111\}$ GB supercells of FCC Fe and $\mathrm{Fe}_{70} \mathrm{TM}_{2}$ alloys. Only the solute atoms in black are shown to highlight their effect zone in figure (c-f). The additional numbers (1 and 2) in (c)-(f) identify the impact of alloying elements (TM) and lattice distortion (LD) on the bond structure of Fe, revealing the chemical and mechanical effects on the electron redistribution. 
(a)
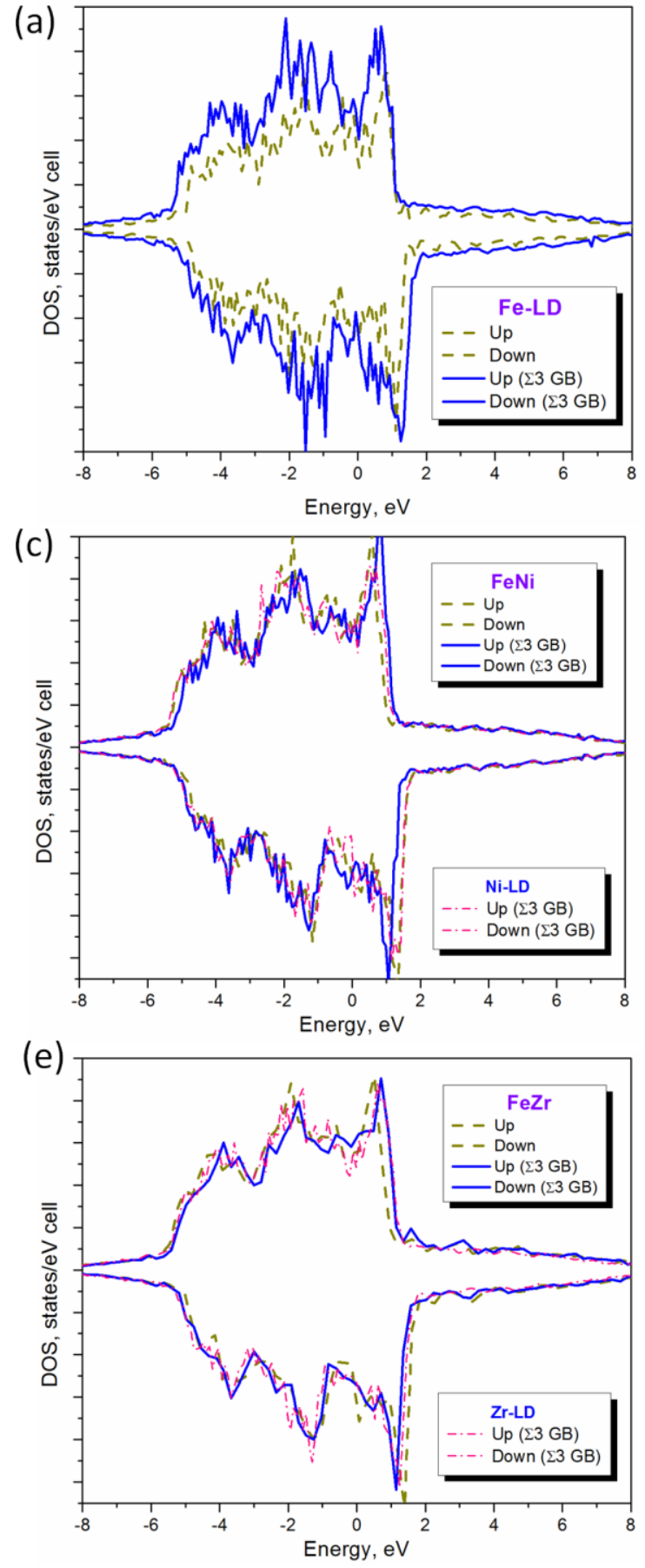

(b)
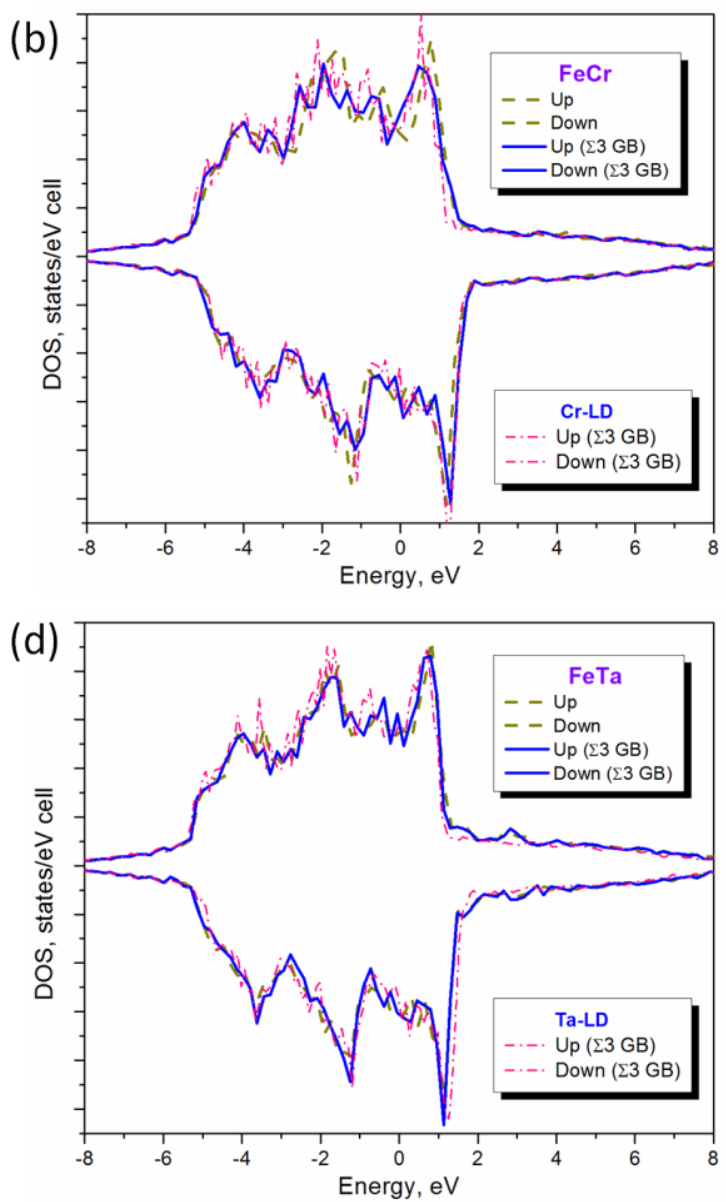

Figure 5 The partial electron density of states (eDOS, including spin $\boldsymbol{U p}$ and spin Down) affected by grain boundary, alloying elements and lattice distortion (LD), (a) pure Fe; (b) Fe-Cr; (c) Fe-Ni; (d) Fe-Ta; and (e) Fe-Zr. The Fermi level defines the zero of energy. 


\section{References:}

[1] Boukhvalov DW, Gornostyrev YN, Katsnelson MI, Lichtenstein Al. Magnetism and Local Distortions near Carbon Impurity in $\gamma$-Iron. Phys. Rev. Lett. 99 (2007) 247205.

[2] Tateno S, Hirose K, Ohishi Y, Tatsumi Y. The Structure of Iron in Earth's Inner Core. Science 330 (2010) 359-361.

[3] Shang S-L, Wang Y, Liu Z-K. Thermodynamic fluctuations between magnetic states from firstprinciples phonon calculations: The case of bcc Fe. Phys. Rev. B 82 (2010) 014425.

[4] Wei BQ, Shima M, Pati R, Nayak SK, Singh DJ, Ma RZ, Li YB, Bando Y, Nasu S, Ajayan PM. Roomtemperature ferromagnetism in doped face-centered cubic Fe nanoparticles. Small 2 (2006) 804809.

[5] Palacios SL, Iglesias R, Martinez-Blanco D, Gorria P, Perez MJ, Blanco JA, Hernando A, Schwarz K. High-temperature anti-Invar behavior of gamma-Fe precipitates in $\mathrm{Fe}_{x} \mathrm{Cu}_{100-x}$ solid solutions: Ferromagnetic phases. Phys. Rev. B 72 (2005) 172401.

[6] Leonov I, Poteryaev Al, Anisimov VI, Vollhardt D. Electronic Correlations at the alpha-gamma Structural Phase Transition in Paramagnetic Iron. Phys. Rev. Lett. 106 (2011) 106405.

[7] Kadau K, Gruner M, Entel P, Kreth M. Modeling structural and magnetic phase transitions in iron-nickel nanoparticles. Phase Transitions 76 (2003) 355-365.

[8] Wang Y, Curtarolo S, Jiang C, Arroyave R, Wang T, Ceder G, Chen LQ, Liu ZK. Ab initio lattice stability in comparison with CALPHAD lattice stability. CALPHAD 28 (2004) 79-90.

[9] Shang SL, Saengdeejing A, Mei ZG, Kim DE, Zhang H, Ganeshan S, Wang Y, Liu ZK. First-principles calculations of pure elements: Equations of state and elastic stiffness constants. Comp. Mater. Sci. 48 (2010) 813-826.

[10] Wang CS, Klein BM, Krakauer H. Theory of Magnetic and Structural Ordering in Iron. Phys. Rev. Lett. 54 (1985) 1852-1855.

[11] van Schilfgaarde M, Abrikosov IA, Johansson B. Origin of the Invar effect in iron-nickel alloys. Nature 400 (1999) 46-49.

[12] Ortiz-Chi F, Aguayo A, de Coss R. Effects of Co doping on the metamagnetic states of the ferromagnetic fcc Fe-Co alloy. J. Phys.: Condes. Matter 25 (2013) 026001.

[13] Keune W, Halbauer R, Gonser U, Lauer J, Williamson DL. Antiferromagnetism of FCC Fe thin-films. J. Appl. Phys. 48 (1977) 2976-2979.

[14] Fernandez-Garcia MP, Gorria P, Blanco JA, Fuertes AB, Sevilla M, Boada R, Chaboy J, Schmool D, Greneche JM. Microstructure and magnetism of nanoparticles with gamma-Fe core surrounded by alpha-Fe and iron oxide shells. Phys. Rev. B 81 (2010) 094418.

[15] Fernandez-Garcia MP, Gorria P, Sevilla M, Fuertes AB, Greneche JM, Blanco JA. Onion-like nanoparticles with $\gamma$-Fe core surrounded by a $\alpha$-Fe/Fe-oxide double shell. J. Alloy. Compd. 509 (2011) S320-S322.

[16] Moruzzi VL, Marcus PM. Magnetism in bcc 3d transition metals: Onset and approach to the Hund's-rule limit. Phys. Rev. B 38 (1988) 1613-1620.

[17] Gebhardt T, Music D, Ekholm M, Abrikosov IA, Vitos L, Dick A, Hickel T, Neugebauer J, Schneider JM. The influence of additions of $\mathrm{Al}$ and Si on the lattice stability of fcc and hcp Fe-Mn random alloys. J. Phys.: Condes. Matter 23 (2011) 246003.

[18] Li XY, Kong LT, Liu BX. Enhanced magnetic moment of Fe in fcc-structured Fe-Ag and Fe-Au alloys synthesized by ion-beam manipulation. Phys. Rev. B 72 (2005) 054118.

[19] Medvedeva NI, Van Aken D, Medvedeva JE. Magnetism in bcc and fcc Fe with carbon and manganese. J. Phys.: Condes. Matter 22 (2011) 316002. 
[20] Yuasa M, Mabuchi M. Effects of segregated $\mathrm{Cu}$ on an Fe grain boundary by first-principles tensile tests. J. Phys.: Condes. Matter 22 (2010) 505705.

[21] Yuasa $M$, Mabuchi $M$. Bond mobility mechanism in grain boundary embrittlement: Firstprinciples tensile tests of Fe with a P-segregated Sigma 3 grain boundary. Phys. Rev. B 82 (2010) 094108.

[22] Kohn W, Sham LJ. Self-consistent equations including exchange and correlation effects. Phys. Rev. 140 (1965) 1133-1138.

[23] Tschopp MA, McDowell DL. Asymmetric tilt grain boundary structure and energy in copper and aluminium. Philos. Mag. 87 (2007) 3871-3892.

[24] Rittner JD, Seidman DN. <110> symmetric tilt grain-boundary structures in fcc metals with low stacking-fault energies. Phys. Rev. B 54 (1996) 6999-7015.

[25] Chadwick G. A., A. SD. Grain boundary structure and properties. Academic Press Inc. (London) LTD (1976).

[26] Couzinie JP, Duparc OH, Lartigue-Korinek S, Thibault-Penisson J, Decamps B, Priester L. On the atomic structure of an asymmetrical near Sigma=27 grain boundary in copper. Philos. Mag. Lett. 89 (2009) 757-767.

[27] Murdoch HA, Schuh CA. Stability of binary nanocrystalline alloys against grain growth and phase separation. Acta Mater 61 (2013) 2121-2132.

[28] Darling KA, Chan RN, Wong PZ, Semones JE, Scattergood RO, Koch CC. Grain-size stabilization in nanocrystalline FeZr alloys. Scripta Mater 59 (2008) 530-533.

[29] Darling KA, Tschopp MA, VanLeeuwen BK, Atwater MA, Liu ZK. Mitigating grain growth in binary nanocrystalline alloys through solute selection based on thermodynamic stability maps. Comp Mater Sci 84 (2014) 255-266.

[30] Kresse G, Furthmuller J. Efficient iterative schemes for ab initio total-energy calculations using a plane-wave basis set. Phys. Rev. B 54 (1996) 11169-11186.

[31] Kresse G, Furthmuller J. Efficiency of ab-initio total energy calculations for metals and semiconductors using a plane-wave basis set. Comp. Mater. Sci. 6 (1996) 15-50.

[32] Kresse G, Joubert D. From ultrasoft pseudopotentials to the projector augmented-wave method. Phys. Rev. B 59 (1999) 1758-1775.

[33] Perdew JP, Chevary JA, Vosko SH, Jackson KA, Pederson MR, Singh DJ, Fiolhais C. Atoms, molecules, solids, and surfaces: Applications of the generalized gradient approximation for exchange and correlation. Phys. Rev. B 46 (1992) 6671.

[34] Wang Y, Perdew JP. Correlation hole of the spin-polarized electron gas, with exact small-wavevector and high-density scaling. Phys. Rev. B 44 (1991) 13298-13307.

[35] Methfessel M, Paxton AT. High-precision sampling for Brillouin-zone integration in metals. Phys. Rev. B 40 (1989) 3616-3621.

[36] Blochl PE, Jepsen O, Andersen OK. Improved tetrahedron method for Brillouin-zone integrations. Phys. Rev. B 49 (1994) 16223-16233.

[37] Birch F. Finite Elastic Strain of Cubic Crystals. Phys. Rev. 71 (1947) 809.

[38] Birch F. Finite strain isotherm and velocities for single-crystal and polycrystalline $\mathrm{NaCl}$ at highpressures and 300 degree K. J. Geophys. Res. 83 (1978) 1257-1268.

[39] Wang WY, Shang SL, Wang Y, Darling KA, Mathaudhu SN, Hui XD, Liu ZK. Electron localization morphology of the stacking faults in Mg: A first-principles study. Chem. Phys. Lett. 551 (2012) 121-125.

[40] Wang WY, Shang SL, Wang Y, Darling KA, Kecskes LJ, Mathaudhu SN, Hui XD, Liu Z-K. Electronic structures of long periodic stacking order structures in Mg: A first-principles study. J. Alloy. Compd. 586 (2014) 656-662. 
[41] Nakashima PNH, Smith AE, Etheridge J, Muddle BC. The Bonding Electron Density in Aluminum. Science 331 (2011) 1583-1586.

[42] Wang WY, Shang SL, Wang Y, Mei Z-G, Darling KA, Kecskes LJ, Mathaudhu SN, Hui XD, Liu Z-K. Effects of alloying elements on stacking faults energies and electronic structures of binary $\mathrm{Mg}$ alloys: A first-principles study. Mater. Res. Lett. 2 (2014) 29-36.

[43] Wang $Y$, Wang WY, Chen L-Q, Liu Z-K. Bonding charge density from atomic perturbations. J. Comput. Chem. 36 (2015) 1008-1014.

[44] Momma K, Izumi F. VESTA: a three-dimensional visualization system for electronic and structural analysis. J. Appl. Crystallogr. 41 (2008) 653-658.

[45] Momma K, Izumi F. VESTA 3 for three-dimensional visualization of crystal, volumetric and morphology data. J. Appl. Crystallogr. 44 (2011) 1272-1276.

[46] Wang Y, Chen LQ, Liu ZK, Mathaudhu SN. First-principles calculations of twin-boundary and stacking-fault energies in magnesium. Scripta Mater. 62 (2010) 646-649.

[47] Pan Z, Kecskes LJ, Wei Q. The nature behind the preferentially embrittling effect of impurities on the ductility of tungsten. Comp. Mater. Sci. 93 (2014) 104-111.

[48] Shang S, Wang Y, Zhang H, Liu Z-K. Lattice dynamics and anomalous bonding in rhombohedral As: First-principles supercell method. Phys. Rev. B 76 (2007) 052301.

[49] Wang Y, Saal JE, Shang SL, Hui XD, Chen LQ, Liu ZK. Effects of spin structures on Fermi surface topologies in BaFe2As2. Solid State Commun. 151 (2011) 272-275.

[50] Shang SL, Wang WY, Zhou BC, Wang Y, Darling KA, Kecskes L, Mathaudhu SN, Liu ZK. Generalized stacking fault energy, ideal strength and twinnability of dilute Mg-based alloys: A first-principles study of shear deformation. Acta Mater 67 (2014) 168-180.

[51] Blaha P, Schwarz K, Dederichs PH. Electronic structure of hcp metals. Phys. Rev. B 38 (1988) 9368-9374.

[52] Manga VR, Saal JE, Wang Y, Crespi VH, Liu Z-K. Magnetic perturbation and associated energies of the antiphase boundaries in ordered Ni3Al. J. Appl. Phys. 108 (2010) 103509.

[53] Antropov VP, Katsnelson MI, van Schilfgaarde M, Harmon BN. Ab Initio Spin Dynamics in Magnets. Phys. Rev. Lett. 75 (1995) 729-732. 